\title{
Postoperative Delirium in Older Inpatients: A survey of Nursing Staff Knowledge
}

\author{
Anant Kumar Verma ${ }^{1}$ \\ ${ }^{1}$ Assistant Professor, Department of Psychiatry, Vardhman Institute of Medical Sciences, Pawapuri, Nalanda-803115, India.
}

\section{Abstract}

Background: Postoperative delirium is common in the elderly and is associated with poor outcome. However, its diagnosis is often missed or delayed. Nursing staff is at the frontline and plays a crucial role in the early detection and management of delirium. This study was designed to explore the knowledge and attitudes of nursing staff about delirium in the scope of an educational program implementation. Subjects and Methods: Qualitative and quantitative analyses conducted in four surgical wards and one intensive care unit in an Academic Hospital in India. A questionnaire was administered to 121 nurses and nursing assistants and semi structured interviews were conducted. Results: A total of 89 questionnaires were completed (response rate of $73.55 \%$ ). Regarding symptoms, most of the nursing staff knew about disorientation and incoherent speech. However, few knew about acute onset and fluctuation, and the hypoactive form of delirium was virtually unknown. Regarding risk factors, while many knew about dehydration, drug use and the use of physical restraints, few knew about sensory impairment and infection. The staff globally knew about the main prevention measures, but knowledge on patient management was especially poor. Finally, no respondent knew about or used the Confusion Assessment Method. The qualitative analysis revealed a trivialization of delirium onset among older inpatients and the continuity of preconceived ideas on delirium, its diagnosis and its risk factors. Conclusion: This study provides a clearer understanding of staff learning needs and identifies potential issues to be addressed in order to increase future intervention efficacy.

Keywords: Delirium; Nursing staff; Knowledge, inpatient.

Corresponding Author: Dr. Anant Kumar Verma, Assistant Professor, Department of Psychiatry, Vardhman Institute of Medical Sciences, Pawapuri, Nalanda-803115, India.

Received: February 2019

Accepted: February 2019

\section{Introduction}

Delirium is a syndrome characterized by fluctuating symptom course of inattention, altered level of consciousness and acute global cognitive impairment. ${ }^{[1]}$ There are three forms of delirium, hypoactive, hyperactive and mixed forms, which fluctuate between the hyperactive and hypoactive phases. ${ }^{[2]}$ The mixed forms account is the most common type (52\%), highlighting the fluctuating course of delirium. ${ }^{[2]}$ The incidence of postoperative delirium in older patients is estimated between 20 and $50 \% .^{[2]}$ Moreover, among older patients admitted to an intensive care unit (ICU), the incidence can reach 70 to $87 \% .^{[1,2]}$ Delirium is associated with a significant increase in morbidity and mortality, a higher risk of admission to a long-term care facility and higher healthcare costs. ${ }^{[3]}$ Although delirium can be reversed through treatment of its underlying causes, many patients do not return to their baseline cognitive level. ${ }^{[2]}$ In addition, in patients with dementia, cognitive decline may be accelerated. ${ }^{[3]}$

Despite its clinical significance, postoperative delirium is frequently under-recognized and misdiagnosed by nurses and physicians. ${ }^{[4-6]}$ Fluctuating changes in cognitive function are commonly misattributed to dementia, depression or old age. ${ }^{[7]}$ Confusion Assessment Method
(CAM) is one of the most sensitive diagnostic tools for detecting delirium [8] and measuring its severity. ${ }^{[9]}$

The nursing staff play a crucial role in the early detection and management of delirium because of their frequent contact with patients and their families. ${ }^{[10,11]}$ The early detection of delirium is critical for the identification of its causes and for the continuous monitoring of its symptoms. ${ }^{[12,13]}$ However, in many patients, delirium is not detected because of the nursing staff's insufficient knowledge on cognitive impairment. ${ }^{[14,15]}$ Nurses' lack of knowledge about the criteria and methods of detecting delirium has been identified as a major factor influencing the under-recognition of delirium. ${ }^{[16]}$ In addition, ageist stereotypes held by many health care professionals appear to represent a major key obstacle in the recognition of delirium. $^{[14]}$

Therefore both knowledge of the nursing staff in terms of delirium recognition and nursing staff's perception of delirium are key factors for improving patients' outcomes. ${ }^{[14,17-20]}$ The goal of this study was to provide an assessment of these parameters prior the implementation of an educational program in order to adapt educational content to staff's educational needs. For a maximum knowledge yield, it was used a mixed method approach in this study, which combine quantitative and qualitative 
data. $^{[21]}$

\section{Subjects and Methods}

\section{Setting}

The present study was conducted in four surgery wards and in one ICU of an Indian academic hospital. The study was reviewed and approved by an ethics committee.

\section{Date collection}

Mixed method survey included a quantitative and qualitative analyses.

\section{Quantitative data}

A questionnaire was developed through an interdisciplinary collaboration assessing nursing staff knowledge on postoperative delirium. It explored four knowledge areas: symptoms, risk factors, diagnostic tools and management. The questionnaire was self administered by nurses and nursing assistants. This questionnaire was also used in the first step of the CONFUCIUS trial, which aims to measure the impact of a multifaceted program on the postoperative delirium prevention in the elderly inpatients. ${ }^{[22]}$

\section{Qualitative data}

The survey questionnaire was complemented by a qualitative approach. ${ }^{[21,23]}$ Field observations were performed during the 18 nursing training sessions. The training sessions were conducted during nursing shift changes and included a total of 72 day staff and 18 night staff. Each standardized session started with an "icebreaker" question, such as, "Tell us about a recently managed patient with postoperative delirium", followed by an open discussion on delirium symptoms and diagnosis. The data were collected from the nursing staff of wards participating in the sudy, starting with the collection of quantitative data.

\section{Data Analysis}

A descriptive analysis of nursing staff characteristics and response rates was performed. Continuous data are presented as mean, and standard deviation (SD) and categorical data are presented as numbers and percentages. Qualitative data were then analyzed by, using a thematic approach to identify recurrent themes. ${ }^{[24]}$ Data were compared across participants.

\section{Results}

A total of 89 questionnaires (52\%) were completed by 53 nurses and 36 nursing assistants. The response rates and characteristics of respondents are shown in [Table 1]. Regarding symptoms, most nurses and nursing assistants knew about disorientation (98\%) and incoherent speech (97\%). However, few knew about acute onset (53\%) and fluctuation (48\%) [Figure 1]. When questioned about behavioral disorders specific to delirium $51 \%$ of the respondents considered agitation an essential feature of delirium, while $45 \%$ considered that agitation/lethargy may alternate. Only $4 \%$ of the respondents stated that an overlethargic and hypoalert behavior could signal delirium as encountered in the hypoactive subtype. Although half of the respondents had work experience in a geriatric ward, geriatric pathologies such as fecaloma and sensory impairment were rarely identified as risk factors of delirium (49\% and 48\%, respectively) [Figure 2]. None of the respondents knew about or used the CAM. Knowledge on patient management was not accurate, especially regarding patient isolation $(6 \%)$ and collaboration with geriatric staff $(8 \%)$. However, the staff globally knew about the main prevention measures: patient reorientation with calendars and clocks (85\%) and patient communication (92\%).

Table 1: Characteristics of the respondents.

\begin{tabular}{|c|c|c|c|c|c|c|}
\hline & Urology & Orthopedics & General Surgery & Oncology & ICU & Overall \\
\hline Respondents, N (\%) & $6(30)$ & $19(44)$ & $12(80)$ & $18(86)$ & $34(43)$ & $89(52)$ \\
\hline Nurse & $1(17)$ & $10(53)$ & $7(58)$ & $13(72)$ & $22(65)$ & $53(60)$ \\
\hline Nursing assistants & $5(83)$ & $9(47)$ & $5(42)$ & $5(28)$ & $12(34)$ & $36(40)$ \\
\hline Work experience in the current position, Years (mean \pm SD) & $23.2 \pm 10$ & $10.5 \pm 10$ & $13.1 \pm 9$ & $12 \pm 11$ & $6.4 \pm 9$ & $10.4 \pm 9$ \\
\hline $\begin{array}{l}\text { Specifically trained on postoperative delirium in the elderly, } \mathrm{N} \\
(\%)\end{array}$ & $0(0)$ & $1(5)$ & $0(0)$ & $7(39)$ & $7(21)$ & $15(17)$ \\
\hline \multicolumn{7}{|l|}{ Professional experience, $\mathrm{N}(\%)$} \\
\hline Geriatrics & $3(50)$ & $8(42)$ & $10(83)$ & $15(83)$ & $16(47)$ & $53(60)$ \\
\hline Neurology & $0(0)$ & $0(0)$ & $5(42)$ & $5(28)$ & $5(15)$ & $15(17)$ \\
\hline Psychiatry & $0(0)$ & $1(5)$ & $7(58)$ & $4(22)$ & $9(26)$ & $22(25)$ \\
\hline
\end{tabular}

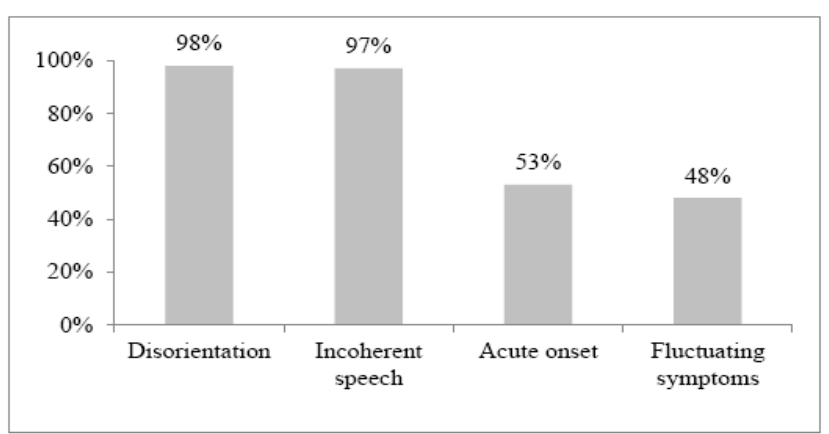

Figure 1: Nursing staff knowledge on the symptoms of delirium

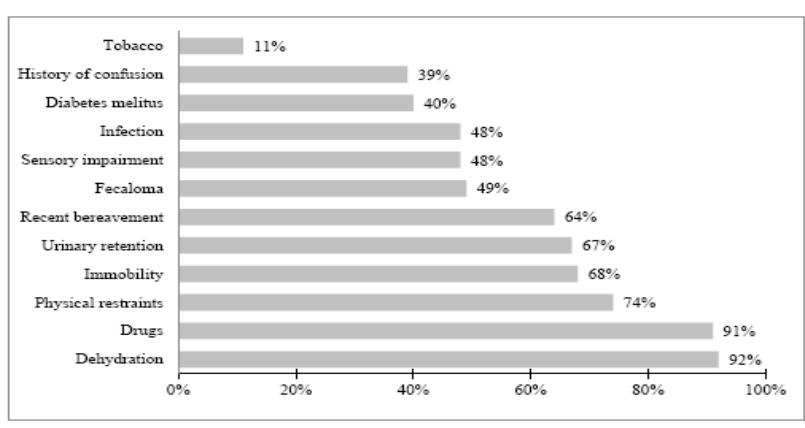

Figure 2: Nursing staff knowledge on the risk factors of delirium 
Three themes were explored during analysis of the semistructured interviews. First, nursing staff knowledge was approached through examples and through the symptoms spontaneously mentioned during the open discussion. A caricature description of postoperative delirium was noted. The examples proposed by the nursing staff often included stereotyped, complicated cases of confusion, with cast, venous or urinary catheter removal, or marked by very odd delusions. The most commonly mentioned symptoms were disorientation, agitated and aggressive behavior and cognitive impairment. Delusions, ambulation, odd speech and day/night fluctuation of symptoms were less often mentioned. Anxiety, behavior modification, and alert by family members were only mentioned once. Unexpected drowsiness and state of coma were not mentioned.

"Do you remember Mister X? He slit his IV line with his teeth!" (A nurse)

"Usually, they ring, they ring... then what they ask for is totally incoherent!" (A nurse)

"First, he became confused, and then walked around in the corridors." (A nurse)

Second, several stereotyped risk factors were presented: medication, especially anesthesia, and evening onset were well-known by the nursing staff. However, other risk factors were rarely mentioned, including pain, benzodiazepine and alcohol withdrawal, hyponatremia and dementia. Other risk factors were never mentioned, including sepsis, urinary and fecal retention, and unfamiliar environment.

"I noticed that people with delusions often have hyponatremia." (A nurse)

"Things are even more difficult when the patients have Alzheimer's disease." (A nursing assistant)

"We think that it is normal... We've been told that after anesthesia, the patients usually rave for a week." (A nurse)

Third, information was gathered about how postoperative delirium in older patients was perceived by the nursing staff. Their description of recent, vivid examples illustrated how postoperative delirium emerged as a problem and disruption in their regular work. Although it was perceived as a complication for the patient, it was something expected and somewhat normal for elderly patients, especially those with dementia. The estimated frequency of episodes differed among nurses, depending on the shift: "maybe once a month" for day shift nurses and "at least once a week" for night shift nurses. The nursing staff declared that they did not receive any training, information or support to handle this condition. However, in one unit, night shift nurses exhibited an informal sheet on a wall, recommending a prescription of hydroxyzine in case of agitation.

"After surgery, the patient became confused; we had a hell of a night!" (A night nurse)

"The patient is anxious, it's strange... We don't know, actually... We imagine that it probably has consequences for the patient." (A nursing assistant, a night nurse)

"Residents on call rarely come when you call them at night..." (A night nurse)

\section{Discussion}

This study allowed us to draw up an inventory of the nursing staff's knowledge and perception of delirium and to assess staff learning needs in order to increase future intervention efficacy.

Our findings indicate that nursing staff working with surgical patients in an Indian academic Hospital have an inadequate level of knowledge base on delirium. We found respondents were not familiar with symptoms suggesting delirium. The hypoactive form of delirium, while accounting for one third of delirium cases, was virtually unknown. In addition, knowledge of risk factors, critical to early detection, was limited and no diagnostic tools were used.

Our results are consistent with the literature showing that nurse's knowledge of delirium is insufficient, particularly regarding risk factors. ${ }^{[17,18]}$ Such limitations can be extended to medical staff since a recent survey have shown that UK training doctors, even though aware of the high prevalence and clinical significance of delirium, had poor knowledge in the diagnosis and management of delirium. ${ }^{[25]}$ It is likely that this knowledge gap in healthcare professionals is a major component of the broadly reported under-recognition of delirium and stems from a historically low educational emphasis on delirium in medical and nursing schools. ${ }^{[26]}$

Consistent with the absence of adequate knowledge, analysis from the semi-structured interviews revealed the persistence of preconceived ideas on delirium, its diagnosis and its risk factors. In particular, most of the staff believed agitation to be an essential feature of delirium. This misconception is widespread and may explain why the hyperactive subtype is still considered the predominant form of delirium, and is best recognized, in spite of its lower prevalence rate. ${ }^{[25]}$

In addition, this lack of knowledge combined in respondents with what appears as a lack of awareness pertaining to the importance of delirium. This was reflected by the responses in the qualitative survey where little or nothing was known about the consequences of delirium on patients' outcome. Moreover, qualitative survey revealed a trivialization of delirium viewed by nursing staff as a consequence of surgery on older inpatients rather than as a preventable condition. Postoperative delirium was perceived by respondents both as a natural occurrence in older patients, and as a time consuming disruption to their regular work. These findings resonate with the results of previous studies suggesting that negative beliefs and attitude are key factors in preventing effective management of older patients with delirium. Overall, our study indicates that healthcare environment in our hospital does not meet the needs of older adults. ${ }^{[19,20,27]}$

The questionnaire used was not subjected to a formal assessment of content validity. There is no previous validated questionnaire to evaluate nursing staff knowledge. This lack of validated questionnaire did not allow us to assess the validity of the questionnaire presented in the 
study. This can be seen as a limitation of the present study. However, the questionnaire was established by a multidisciplinary group of healthcare professionals. Another limitation is that only the knowledge of the nursing staff in four surgical wards and in one ICU was assessed. Therefore, the results must be restricted to surgical nursing staff.

\section{Conclusion}

Thus it is found that there is an urgent need for educational emphasis on delirium in medical and nursing schools. Given the prevalence of delirium among older patients, a change in hospital culture as well as in health care provider's training is needed.

\section{References}

1. Fong TG,Tulebaev SR, Inouye SK(2009) Delirium in elderly adults: diagnosis, prevention and treatment. Nat Rev Neurol 5: 210-220.

2. Inouye SK(2006) Delirium in Older Persons. New England Journal of Medicin 354:1157-1165.

3. Witlox J, Eurelings LS, de Jonghe JF, Kalisvaart KJ, Eikelenboom P, et al.(2010) Delirium in Elderly Patients and the Risk of Postdischarge Mortality, Institutionalization, and Dementia. JAMA: The Journal of the American Medical Association 304: 443-451.

4. Inouye SK, Foreman MD, Mion LC, Katz KH, Cooney LM Jr(2001) Nurses' Recognition of Delirium and Its Symptoms: Comparison of Nurse and Researcher Ratings. Arch Intern Med 161: 2467-2473.

5. Young LJ, George J (2003) Do guidelines improve the process and outcomes of care in delirium? Age and Ageing 32: 525-528.

6. Gustafson Y, Brännström B, Norberg A, Bucht G, Winblad B(1991) Underdiagnosis and poor documentation of acute confusional states in elderly hip fracture patients. J Am GeriatrSoc 39: 760-765.

7. Fick DM, Hodo DM, Lawrence F, Sharon KI(2007) Recognizing delirium superimposed on dementia: assessing nurses' knowledge using case vignettes. J GerontolNurs 33: 40-47; quiz 48-9.

8. Fick DM, Agostini JV, Inouye SK (2002) Delirium Superimposed on Dementia: A Systematic Review. Journal of the American Geriatrics Society 50: 1723-1732.

9. De J, Wand AP (2015) Delirium Screening: A Systematic Review of Delirium Screening Tools in Hospitalized Patients. Gerontologist 55: 1079-1099.

10. Schuurmans MJ, Deschamps PI, Markham SW, Shortridge-Baggett LM, Duursma SA(2003) The measurement of delirium: review of scales. Res Theory NursPract 17: 207-224.

11. Faught DD (2014) Delirium: The Nurse's Role in Prevention, Diagnosis, and Treatment.MedsurgNurs 23: 301-305.

12. Foreman $\mathrm{MD}(1986)$ Acuteconfusional states in hospitalized elderly: a research dilemma.Nurs Res 35: 34-38.

13. Steis MR, Fick DM(2008) Are nurses recognizing delirium? A systematic review. J GerontolNurs 34: 40-48.
14. Milisen K, Steeman E, Foreman MD(2004) Early detection and prevention of delirium in older patients with cancer. European Journal of Cancer Care 13: 494-500.

15. Wells Y, Foreman P, Gething L, Petralia W (2004) Nurses' attitudes toward aging and older adults--examining attitudes and practices among health services providers in Australia. J GerontolNurs 30: 5 13.

16. Eden BM, Foreman MD(1996) Problems associated with underrecognition of delirium in critical care: A case study. Heart \& Lung: The Journal of Acute and Critical Care 25: 388-400.

17. Hare M, Wynaden D, McGowan S, Landsborough I, Speed G (2008) A questionnaire to determine nurses' knowledge of delirium and its risk factors.Contemp Nurse 29: 23-31.

18. Meako ME, Thompson HJ, Cochrane BB (2011)Orthopaedic nurses' knowledge of delirium in older hospitalized patients.OrthopNurs 30 : 241-248.

19. McCarthy MC (2003) Situated clinical reasoning: Distinguishing acute confusion from dementia in hospitalized older adults. Research in Nursing \& Health 26: 90-101.

20. McCarthy MC (2003) Detecting acute confusion in older adults: Comparing clinical reasoning of nurses working in acute, long-term, and community health care environments. Research in Nursing \& Health 26: 203-212.

21. O'Cathain A, Murphy E, Nicholl J (2007)Why, and how, mixed methods research is undertaken in health services research in England: a mixed methods study. BMC Health Serv Res 7: 85.

22. Mouchoux C, Rippert P, Duclos A, Fassier T, Bonnefoy M, et al. (2011) Impact of a multifaceted program to prevent postoperative delirium in the elderly: the CONFUCIUS stepped wedge protocol. BMC Geriatr 11: 25.

23. Malterud K, (2001) Qualitative research: standards, challenges, and guidelines. Lancet 358: 483-488.

24. Pope C, Ziebland S, MaysN (2000) Qualitative research in health care. Analysing qualitative data.Bmj 320: 114-116.

25. Davis D, MacLullich A (2009) Understanding barriers to delirium care: a multicentre survey of knowledge and attitudes amongst UK junior doctors. Age and Ageing 38: 559-563.

26. Afriyie-Boateng M, Loftus C, Hamelin MA (2015) Use of a Multimodal Implementation Strategy to Improve Delirium Screening by Nurses on an Acute Care for Elders Unit. Worldviews Evid Based Nurs.

27. Dahlke S, Phinney A(2008) Caring for hospitalized older adults at risk for delirium: the silent, unspoken piece of nursing practice. J GerontolNurs34: 41-47.

28. Marcantonio ER (2012) Postoperative delirium: a 76-year-old woman with delirium following surgery. JAMA 308: 73-81.

29. Rockwood K, Cosway S, Stolee P, Kydd D, Carver D, et al. (1994) Increasing the recognition of delirium in elderly patients. Journal of the American Geriatrics Society 42: 252-256.

30. Tabet N, Hudson S, Sweeney V, Sauer J, Bryant C, et al. (2005) An educational intervention can prevent delirium on acute medical wards. Age Ageing 34: 152-156.

31. Wand AP, Thoo W, Sciuriaga H, Ting V, Baker J, et al.(2014) A multifaceted educational intervention to prevent delirium in older inpatients: a before and after study.Int J Nurs Stud 51: 974-982.

Copyright: (C) the author(s), 2019. It is an open-access article distributed under the terms of the Creative Commons Attribution License (CC BY 4.0), which permits authors to retain ownership of the copyright for their content, and allow anyone to download, reuse, reprint, modify, distribute and/or copy the content as long as the original authors and source are cited.

How to cite this article: Verma AK. Postoperative Delirium in Older Inpatients: A survey of Nursing Staff Knowledge. Asian J. Med. Res. 2019;8(1):PY01-PY04.

DOI: dx.doi.org/10.21276/ajmr.2019.8.1.PY1 